\title{
Disturbance of Information in Superior Parietal Lobe during Dual-task Interference in a Simulated Driving task
}

Mojtaba Abbas-Zadeh¹, Gholam-Ali Hossein-Zadeh ${ }^{1,2}$, Shima Seyed-Allaei ${ }^{1}$, Maryam Vaziri-Pashkam ${ }^{3}$

${ }^{1}$ School of Cognitive Sciences, Institute for Research in Fundamental Sciences, Niavaran, Tehran, Iran

${ }^{2}$ School of Electrical and Computer Engineering, College of Engineering, University of Tehran, Tehran, Iran

${ }^{3}$ Laboratory of Brain and Cognition, National Institute of Mental Health, Bethesda, Maryland, USA

Corresponding Author: Mojtaba Abbas-Zadeh

Address: School of Cognitive Sciences, Institute for Research in Fundamental Sciences (IPM), Artesh Highway, Aghdassieh, Tehran, Iran

Phone: (+98) 2126113274, Email: mabbaszadeh@ipm.ir

Conflict of interest: The authors declare no conflicts of interest. 


\section{Abstract}

Performing a secondary task while driving causes a decline in driving performance. As an important case of dual-task interference, this may generate lethal consequences. Previous investigations on the neural correlates of dual-task interference used simple and artificial stimuli. The neural mechanism of this effect in real-world tasks such as driving, is not yet fully understood. Using fMRI, we aimed to investigate the neural underpinnings of dual-task interference in driving. Participants performed a lane change task in a simulated driving environment, along with a tone discrimination task with either short or long time onset difference (Stimulus Onset Asynchrony, SOA) between the two tasks. Behavioral results indicated a strong dual-task effect on driving reaction times. The univariate analysis of $\mathrm{fMRI}$ data uncovered the modulation of the HRF in the sensory, central, and motor regions of the brain across different SOA conditions. To investigate the effect of dual-task interference on the spatial pattern of brain activity in the regions involved in driving, we used multi-voxel pattern analysis (MVPA) with a linear classifier to decode driving directions. Above chance decoding accuracies were observed in visual and motor regions as well as a central superior parietal lobe (SPL). Comparing accuracies across SOAs, no effect of SOA on accuracies was observed in the visual and motor regions. The SPL region, however, showed a drop in decoding accuracy in short compared to long SOA. Also, the classification accuracy in this region was inversely correlated with participants' reaction time in the driving task. These results suggest a direct link between the information content of the central region SPL and dual-task interference in a naturalistic simulated driving task. 
bioRxiv preprint doi: https://doi.org/10.1101/2020.07.28.224394; this version posted July 29, 2020. The copyright holder for this preprint (which

was not certified by peer review) is the author/funder, who has granted bioRxiv a license to display the preprint in perpetuity. It is made available under aCC-BY-NC-ND 4.0 International license.

Keywords: Dual-task interference, Driving, Time-resolved fMRI, Multi-voxel Pattern Analysis 


\section{Introduction}

Performing a secondary task during driving causes a decline in driving performance (i.e., Hibberd, Jamson, \& Carsten, 2013; Levy, Pashler, \& Boer, 2006). Especially, when time lag between the onsets of driving task and the secondary task (henceforth referred to as the Stimulus Onset Asynchrony, SOA) decreases, reaction times and the accuracies are deteriorated. This decline in performance as a function of SOA is used as a measure of dual-task interference (Pashler, 1994). Although dual-task interference has been widely studied using behavioral techniques, its underlying neural mechanisms are not well understood. In this study, we explored underlying neural mechanisms of dual-task interference using $\mathrm{fMRI}$ and a well-controlled simulated driving task. To find brain regions that could account for behavioral decline during dual-task interference, we investigated changes in average activity as well as information content of multiple cortical regions across short and long SOA conditions.

Most previous dual-task studies have used artificial dual-task paradigms for characterizing source of interference (Dux, Ivanoff, Asplund, \& Marois, 2006; Hesselmann, Flandin, \& Dehaene, 2011; Jiang, Saxe, \& Kanwisher, 2004; Nijboer, Borst, van Rijn, \& Taatgen, 2014; Sigman \& Dehaene, 2008). Based on these studies, it is not clear whether identical neural regions are also associated with the decline in performance during real-world conditions such as performing a secondary task while driving. A dualtask paradigm similar to a real-world situation in which the parameters in traditional tasks are still finely controlled might likely evoke mental states more typical to the ones seen in real-world situations. A few studies have investigated brain regions involved in dual-task 
interference in a simulated driving environment by comparing dual-task with single-task conditions (i.e.(Al-Hashimi, Zanto, \& Gazzaley, 2015; Just, Keller, \& Cynkar, 2008)) and they reported modulations in the activity of the parietal and frontal cortices. Interpreting findings from these studies is difficult, as the single-task condition is not a clean control for isolating the dual-task effect. For isolating the dual-task effect, it is better to compare short SOA and long SOA conditions, as these two conditions are only different in timing between the two tasks. The manipulation of SOA provides the possibility to localize regions that their activation correlates with the magnitude of dual-task interference (Herath et al., 2005). Therefore, regions isolated by comparing short and long SOA conditions are potentially more specific than regions isolated by comparing dual- and single-task conditions. In this study, we used this approach to isolate regions affected during dual-task interference.

Previous fMRI studies of dual-task, including ones that have used simple tasks as well as driving studies, have focused on univariate methods for investigating neural correlates of dual-task interference, looking at either the peak or time-course of the BOLD response. From these results, it is not clear whether overall modulations in brain activity is a result of general effects such as task difficulty, attentional modulations, and mental effort, or it is caused by a specific change in neural responses to each condition due to interference in perceptual and cognitive information. To overcome this limitation, in addition to univariate analyses, we investigated changes in patterns of responses of brain regions in short compared to long SOAs. To the best of our knowledge, there is no fMRI study that has used multivariate methods (e.g., Cox and Savoy (2003)) to investigate neural underpinnings of dual-task interference. 
In sum, two main questions were addressed in the current study. First, is there any brain region that its mean activity changes in short compared to long SOA conditions? Second, is there any brain region in which the information for driving direction is disrupted in short compared to long SOA conditions? We used a dual-task paradigm in which a driving task was performed concurrently with a tone discrimination task in two SOA conditions. This paradigm allows us to investigate how response in various brain regions changes across the time when two tasks tightly compete with each other for the resources.

\section{Material and methods}

\section{Participants}

Twenty-four right-handed volunteers (16 females), 20-36 years old with normal or corrected-to-normal vision and no history of neurological or psychiatric disorders participated in the experiment. Participants gave informed consent and received payment for their participation. The ethics committee at the Institute for Research in Fundamental Sciences (IPM) approved the experiment. Four participants were excluded from the analysis due to excessive head movement $(>5 \mathrm{~mm})$ during the scan and the results of 20 participants (12 females) were analyzed.

\section{Experimental design and procedure}

Apparatus: Stimuli were presented on a 32 " monitor placed at the back of the fMRI scanner bore and viewed via a head coil-mounted mirror. Participants responded to the tasks using two fMRI compatible Current Designs four button response pads, one for each hand. They responded to the driving task with their left index and middle fingers and to the tone task with their right index and middle fingers. 
Stimuli and Paradigm: The fMRI dual-task paradigm consisted of a driving lane change task and a tone discrimination task. The Unity 3D game engine was used to design the driving environment. The driving environment included a highway with infinite lanes on the two sides, without left/right turns or inclining/declining hills. This was to equalize all trials in terms of visual appearance for the purpose of the experiment (see Figure 1). The driving stimulus was composed of two rows of traffic cones with three cones in each row (Figure 1). In each trial, traffic cones were unexpectedly displayed on both sides of one of the lanes, and the participants had to steer the vehicle immediately to the lane with the cones and drive through them. The distance between the two rows of cones was such that the vehicle could easily drive through them without collisions. The cones were always presented immediately to the left or the right lane so that the participants had to change only one lane per trial. The lane change was performed gradually, and the participants had to hold the corresponding key to direct the vehicle in between the two rows of cones, and then release the key when the vehicle was situated correctly. Any collision with the cones would be registered as an error. The fixation cross was jittered for $100 \mathrm{~ms}$ to provide online feedback in case of a collision with the traffic cones.

The driving started with a given initial speed which was kept constant. During the experiment, the participant moved to the right or left lanes by pressing the keys using the middle and index fingers of their left hand, respectively. For the tone task, a single pure tone of either a high $(800 \mathrm{~Hz})$ or low $(400 \mathrm{~Hz})$ frequency was presented for 200 milliseconds. Participants pressed the keys with the middle and index fingers of their right hand to determine whether the tone was a high frequency or a low frequency, respectively. To provide feedback, if participants responded incorrectly, the green fixation 
cross turned red. The onset of each trial was set to be the presentation time of tone stimulus and the end of each trial was set to be when the rear end of the car reached the end of the set of traffic cones. Participants were told to focus on the fixation cross at the center of the page and respond as fast as possible to each task that was presented. The performance in the driving task was calculated as the percentage of trials in which the participant passed through the cones without collision. The performance in the tone task was calculated as the number of correct identifications.

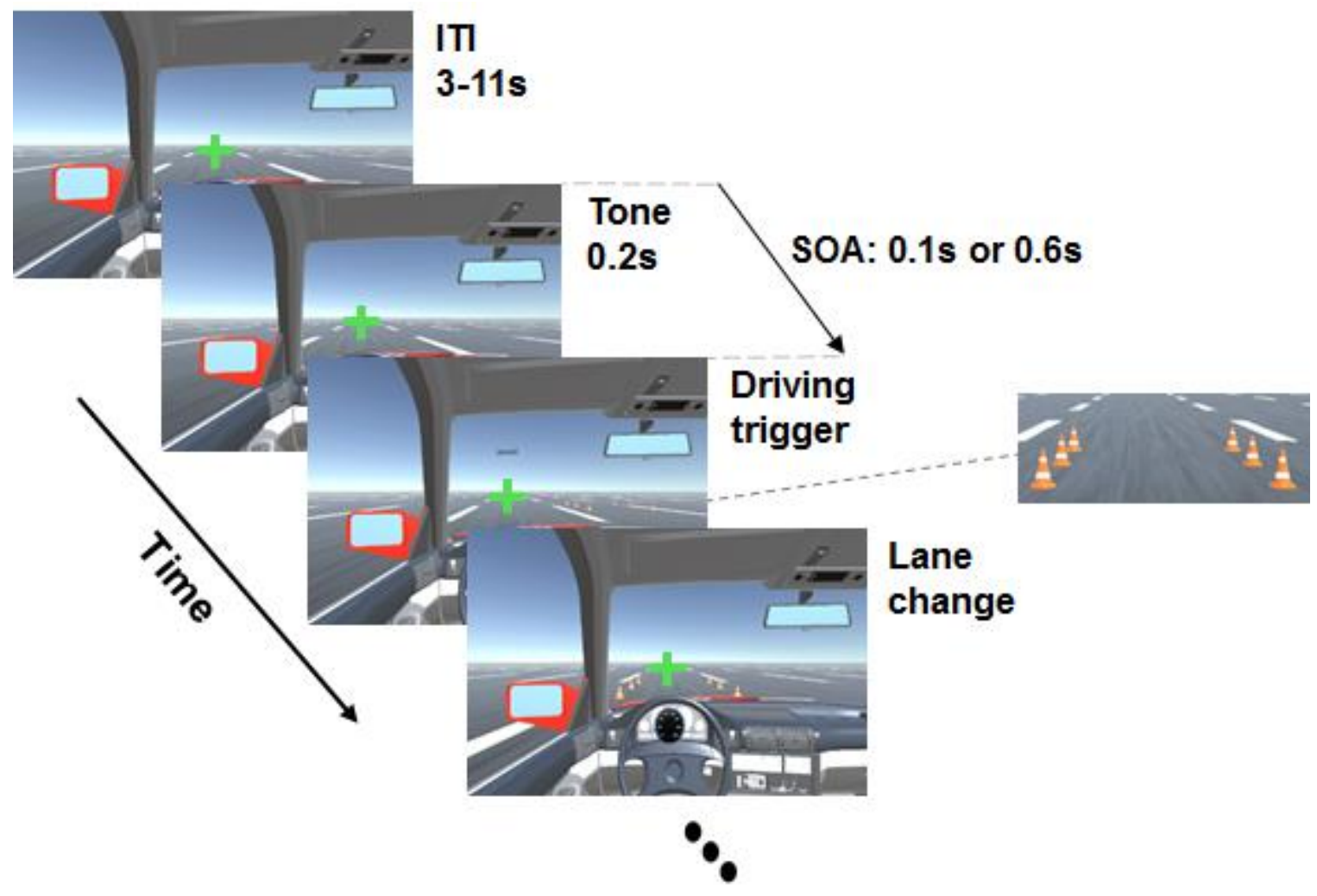

Figure 1. The sequence of events for a sample trial of dual-task paradigm. The Inter trial interval (ITI) lasted between 3 to $11 \mathrm{~s}$. The tone lasted for $200 \mathrm{~ms}$ and the driving trigger was presented 100 or 600 after the tone stimulus. Participants had to perform a tone discrimination task immediately after the presentation of the tone and a lane change immediately after the driving stimulus (two rows of cones). 
The experiment consisted of the dual-task and single-task conditions. In the dual-task trials, the two tasks were presented with either a short $(100 \mathrm{~ms})$ or long $(600 \mathrm{~ms})$ SOAs. In the single-task trials either the driving or the tone task was presented alone. There were a total of eight dual-task conditions: two SOAs $x$ two driving directions (turn right/turn left) $x$ and two tone conditions (low/high frequency); and four single-task conditions: two directions $\mathrm{x}$ two tone conditions. In the dual-task conditions the order of the presentation of tasks was fixed so that the tone task was always presented first and the driving task was presented second. Each condition was repeated four times in each run resulting in a total of 48 trials in every run. Each trial lasted for $3 s$ with an inter-trial interval varying from 3s to 11s. We used optseq software (Dale, 1999) for optimizing the presentation order of trials in each run. The participants completed 12 runs and each lasting $4.8 \mathrm{~min}$ (288 sec). In all dual-task trials the tone task was presented first. Before performing the main experiment, all participants performed two training runs similar to the main experiments. If their performance was $80 \%$ or higher, they would proceed to the main experimental runs. All participants could reach this threshold.

\section{Image acquisition}

Structural and functional images were acquired using a Prisma Siemens 3T MRI scanner at National Brain Mapping Laboratory with a 64 channel head coil for 4 of the participants and a Tim Trio Siemens 3T MRI scanner with 32 channel head coil at the IPM Imaging Center for all other participants. The IPM Imaging Center scanner was originally unavailable due to technical reasons, therefore we started the experiments at the National Brain Mapping Laboratory. After the IPM scanner became available we switched to collecting data at IPM. The imaging parameters were kept the same across scanners 
and the results were similar. T1-weighted images were acquired with gradient echo sequence with $1900 \mathrm{~ms}$ repetition time $(\mathrm{TR})$, echo time $(\mathrm{TE})=2.52$, field of view $(\mathrm{FOV})=$ $256 \mathrm{~mm}$, matrix size of $256 \times 256$, with 192 slices with $1 \mathrm{~mm}$ thickness, iso voxel size of $1 \mathrm{~mm}$, and flip angle of 9 degrees. These high-resolution images were used for surface reconstruction. Blood oxygenation level-dependent (BOLD) functional images were acquired using a single-shot gradient EPI sequence with TR $=2000 \mathrm{~ms}$, TE $=26 \mathrm{~ms}$, flip angle $=90$ degree, matrix size of $64 \times 64, \mathrm{FOV}=192 \mathrm{~mm}, 33$ slices with $0.3 \mathrm{~mm}$ slice gap, and voxel size of $3 \times 3 \times 3 \mathrm{~mm}$.

\section{Image Preprocessing}

Initial image analysis was performed using the Freesurfer image analysis suite, (http://surfer.nmr.mgh.harvard.edu/) and fsfast (Dale, Fischl, \& Sereno, 1999). Pattern classification was performed using CoSMoMVPA toolbox in MATLAB (Oosterhof, Connolly, \& Haxby, 2016) and in-house MATLAB code. FMRI preprocessing included 3D motion correction, slice timing correction and linear and quadratic trend removal. For the whole brain group-level analysis, the anatomical T1-weighted images of each participant were transformed into standardized Freesurfer fsaverage space (Evans et al., 1993). For GLM analysis data were spatially smoothed with a Gaussian kernel of $6 \mathrm{~mm}$ FWHM for univariate analysis, but non-smoothed data were used for multivariate analysis. For every condition, we used a finite impulse response (FIR) basis function (Henson, Rugg, \& Friston, 2001) to examine the changes of BOLD response across time. For this purpose, the BOLD response was quantified for 10 post-stimulus time bins with each time bin representing one full-brain volume of $2 \mathrm{~s}$ duration. These time bins were time-locked to 
the onset of the tone stimulus in the dual-task trials and with the onset of the tone stimulus or the driving cones in the single-task tone and driving trials, respectively.

\section{Univariate analysis}

A general linear model was used to estimate beta values for each vertex and each FIR time bin. As mentioned in the previous section, there were 10 post-stimulus FIR time bins that were time-locked to the onset of the stimulus. For the GLM model, we used twelve regressors of interest: eight for the dual-task conditions (two driving lane change directions $x$ two tone types $x$ two SOAs) and four for the single-task conditions (two driving lane change direction and two tone types). The focus of the current study was to compare the short and long SOA conditions; therefore, the result of single-task conditions was excluded from the analysis in this paper. To compare the mean activity of short and long SOA conditions, we contrasted the short versus long SOA conditions in time bins 2 to 4 to generate a contrast map for each participant and each time bin.

These maps were used to create regions of interest. To avoid double-dipping, we used a leave-one-subject-out procedure (Esterman, Tamber-Rosenau, Chiu, \& Yantis, 2010; Loose, Wisniewski, Rusconi, Goschke, \& Haynes, 2017). For this procedure, we left one participant out and performed the group analysis on all other participants to find clusters that show a significant difference in activity between the two SOA conditions. This procedure was performed for each participant in time bins 2 to 4 . The output was three p-value maps for each participant. Using a union analysis, we calculated a p-value map equal to the minimum $p$-value across the three FIR time bins. Next, the union p-value map was corrected for multiple comparisons using cluster-extent thresholding with $p<$ 
0.001 at the voxel level and $p<0.05$ at the cluster level. The significant clusters on the 19 participants were selected as ROls for the left-out 20th participant. We repeated this procedure, leaving one participant out at a time, to create independent ROls for all participants. Finally, we compared the mean activity for short and long SOA conditions in all FIR time bins in these ROls. The left and right hemispheres ROls were merged for all ROls that were in two hemispheres. To statistically compare the mean activation of each ROI for short and long SOAs, we ran a two-way repeated measure ANOVA with SOA (short and long) and FIR time bins as two factors and p-values were corrected for multiple comparisons with False Discovery Rate (FDR,(Benjamini \& Hochberg, 1995) q < 0.05 across the ROls. The paired t-test was used to compare the mean activity for the short and long SOA conditions in each ROI and each time bin. The p-values were corrected for multiple comparisons with FDR $q<0.05$ across time bins for each ROI.

\section{Multivariate pattern analysis (MVPA)}

In this analysis, we assessed whether information about the driving direction is encoded differently in the short and long SOA conditions. First, using a searchlight approach we identified the regions that coded the driving direction in either the short SOA or the long SOA conditions. Next, we quantified the differences between the decoding accuracies in the short and long SOA conditions in an ROI analysis.

Using CoSMoMVPA ((Oosterhof et al., 2016)), we ran a surface-based searchlight analysis (Oosterhof, Wiggett, Diedrichsen, Tipper, \& Downing, 2010) on the beta maps of FIR time bin 3 (the peak hemodynamic time-bin) for each individual participant. The surface-based searchlight was restricted to a mask containing only voxels between the 
pail and white surfaces of the brain. The pail and white surfaces were generated using Freesurfer. Using the pial and white surfaces an intermediate surface was estimated which placed between two surfaces. A center vertex on the intermediate surface was selected and then 100 neighboring voxels around the center vertex were chosen based on the geodesic distance as a region. The procedure was performed for all vertices on the intermediate surface that included the entire volume of the gray matter of the brain. The estimated beta value patterns were extracted in every region. Then, in each region, the presence of information about the driving direction was assessed by a linear support vector machine classifier. A leave-one-run-out cross-validation procedure was used to evaluate the classification performance (Kamitani \& Tong, 2005). The classifier was trained to discriminate between the two classes (turn left vs turn right) from all but one run and tested on the left-out run. This process was repeated for the 12 runs and the resulting performances were averaged to generate the mean classification accuracy for each searchlight center vertex. This analysis was performed separately for the short and long SOA conditions to produce a whole-brain classification accuracy map for each participant and each SOA condition. The average accuracy maps were normalized to a common space (fsaverage). A Gaussian kernel with $6 \mathrm{~mm}$ full-width at half maximum was used to smooth the accuracy maps.

To compare the information of the driving task in the short and long SOAs, we performed an ROI analysis using the accuracy maps of the short and long SOA. To define the ROIs, we performed a leave-one-subject-out procedure (Esterman et al., 2010) to avoid doubledipping (Kriegeskorte, Simmons, Bellgowan, \& Baker, 2009). One participant was left out and by using the remaining participants, a group-level analysis was performed to find 
clusters that their decoding accuracies were significantly above chance separately for short and long SOAs. Then using a union analysis, we obtained a p-value map equal to the minimum $p$-value of the short and long SOA. This $p$-value map was then corrected for multiple comparisons with voxel-wise $p<0.001$ and cluster thresholded at $p<0.05)$. These clusters related to the 19 participants were selected as ROls for the left out 20th participants and the procedure was repeated to obtain independent ROls for all participants. The mean accuracies across SOAs were compared in these ROls. To statistically compare the mean accuracy of each ROI for short and long SOAs, we ran a two-way repeated measure ANOVA with SOA (short and long) and FIR time bins as two factors and $p$-values were corrected for multiple comparisons with FDR $q<0.05$ across the ROls. The comparison of $p$-value with the chance level $(50 \%)$ was performed by a one-sample t-test for each $\mathrm{ROI}$ and each time bin. The pairwise comparison of accuracy for the short and long SOA conditions was performed by paired t-test in each ROI and each time bin. The $p$-values were corrected for multiple comparisons with FDR $q<0.05$ across time bins for each ROI.

\section{Results}

\section{Behavioral results}

We compared the mean RT and accuracy across long and short SOA conditions for the driving and the tone task (Figure 2). For the driving task which was always presented second, mean RT was significantly greater in the short SOA trials than the long SOA trials $(t(19)=-13.23, p<0.0001$, paired t-test). Although the participants had high accuracy in the driving task (accuracy > 95\%), the participants' accuracy was significantly lower in 
short compared to long SOA condition $(t(19)=-4.06, p<0.001$, paired $t$-test). For the tone task, the RT did not significantly change SOA conditions ( $p s>0.05)$, while the accuracy was significantly lower in the short compared to the long SOA trials $(t(19)=$ 4.11, $p<0.001$, paired t-test). These findings show that the performance of two tasks influenced by dual-task interference, although the effect is stranger for the reaction time of driving task $(t(19)=-4.63, p<0.0001$, paired t-test). These findings are consistent with previous studies (Abbas-Zadeh, Hossein-Zadeh, \& Vaziri-Pashkam, 2019; Hibberd et al., 2013) and indicate a strong effect of dual-task interference.
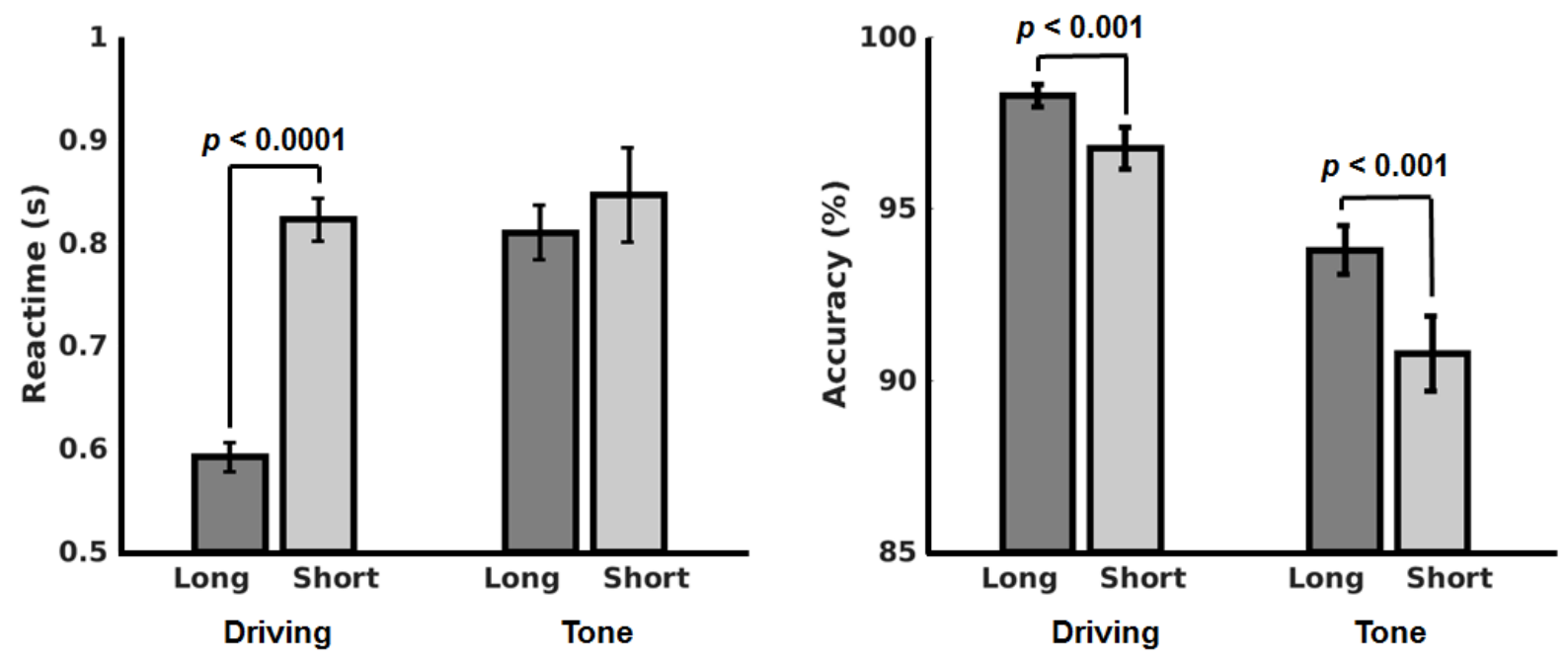

Figure 2. A) Reaction times and B) Accuracies for the short SOA (dark gray bars) and long SOA (light gray bars) conditions in the driving and the tone tasks. Barplots and errorbars show the mean and standard error, respectively, for each condition.

\section{Univariate Analysis}

To investigate the changes of BOLD response during dual-task interference, we performed a univariate analysis to explore whether any brain region shows a different activation in the short compared to long SOA conditions across time. For this purpose, we performed a time-resolved FIR analysis. The beta maps related to FIR time bins 2 to 
4 (corresponding to $2-8 \mathrm{~s}$ from the start of the trial) were used to extract the regions that their activity changed in the short compared to the long SOA condition.

Using a union analysis, we extracted all regions that showed a significant change of activation in the short compared to the long SOA condition in any of the time bins 2 to 4. To avoid non-independence error, we used a leave-one subject-out procedure (see methods, (Esterman et al., 2010; Loose et al., 2017) to extract the mean activation of ROls in selected time bins for the short and long SOA conditions. The overlay of ROls for all participants that were created with the leave-one-subject-out procedure is shown in Figure 3. Each subplot shows the change of beta values across time bins for the short and long SOA conditions. The ROIs that showed modulation of activity in two SOA conditions could be divided into three groups: 1) sensory regions (bilateral visual cortex and bilateral auditory cortex); 2) decision and response selection related regions (bilateral inferior frontal gyrus (IFG), left inferior frontal junction (IFJ); left medial frontal gyrus, bilateral Insula, bilateral superior frontal gyrus, bilateral cingulate cortex); 3) motor planning and execution regions (bilateral precentral and postcentral gyri).

To statistically investigate the temporal changes of BOLD response in the short and long SOA conditions in every ROI, we ran a two-way repeated-measure ANOVA with SOA (short and long) and FIR time bins (time bins 1 to 10) as two factors. The details of statistical testing for all ROls are reported in table 1. The main effect of SOA was only significant for the bilateral visual cortex $(F(1,19)=23.51, p=0.002$, two-way repeatedmeasures ANOVA). However, the interaction of SOA and FIR time bins was significant for all regions ( $p s<0.0001$, corrected for multiple comparisons across ROls). Using paired t-test, we also compared the BOLD response in each FIR time bin across short 
and long SOAs for all ROls and details of statistical testing can be seen in table 2 . The results showed that in most regions, the difference between short and long SOAs for time bins 4 and 5 was significant $(p s<0.05$, corrected for multiple comparisons across time bins). As it is evident in the subplots of Figure 3, although for most of the regions the third FIR time bin is the peak of the hemodynamic response function (HRF) for both short and long SOA conditions, the difference between conditions manifests in the time bin after the peak of the response, where the BOLD response is higher for the short compared to the long SOA condition ( $t s>4.21$, ps $<0.005$, paired-test). In addition, the results revealed a delay in the rise of the HRF (lower response in the time bin before the peak) in the short SOA compared to the long SOA in the auditory, precentral, postcentral and posterior part of superior medial frontal regions ( $t s<-3.46, p s<0.017$, paired-test). The results indicate that the dual-task interference causes the change in the shape of the BOLD signal rather than the change in the peak of the BOLD signal in most modulated ROls. 
L IFJ

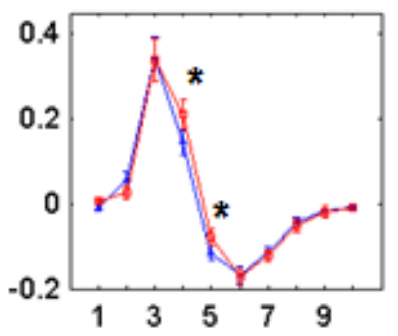

\section{L dMFC}

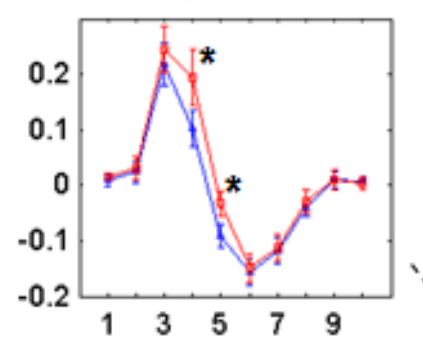

L Supramarginal
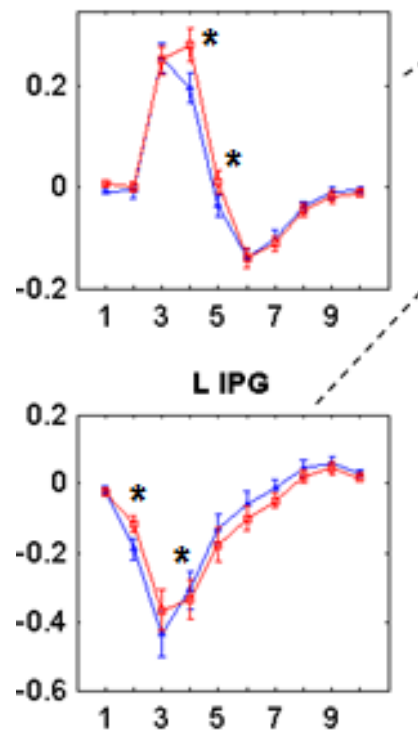

Visual

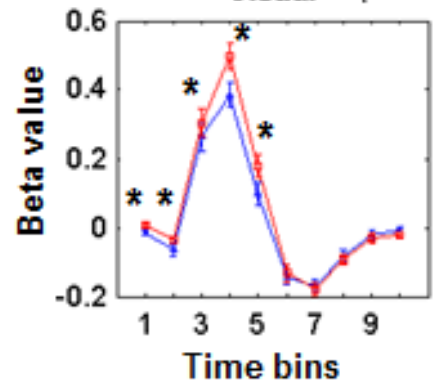

Postcentral

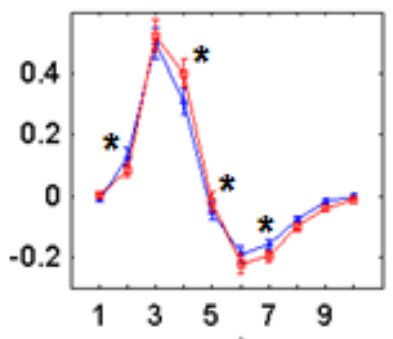

$-0.2$

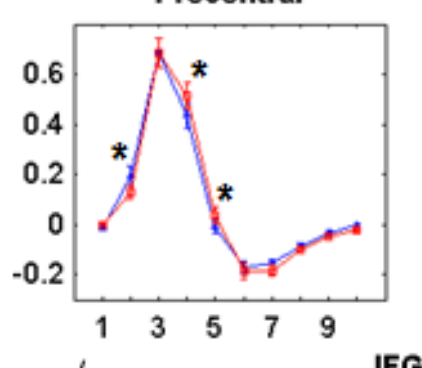

FG
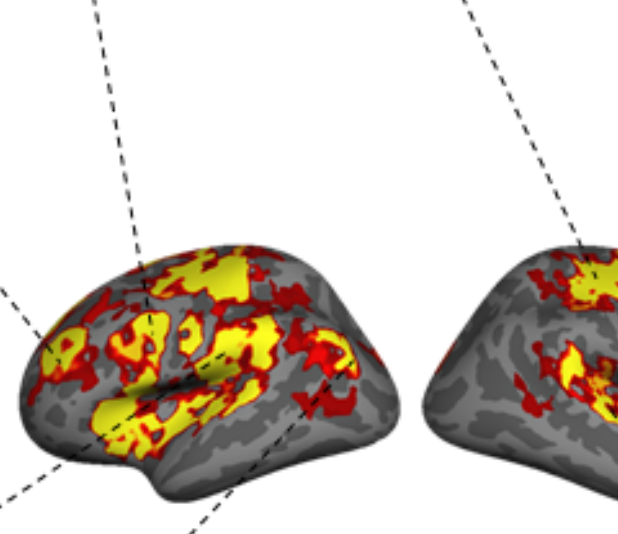

0.2
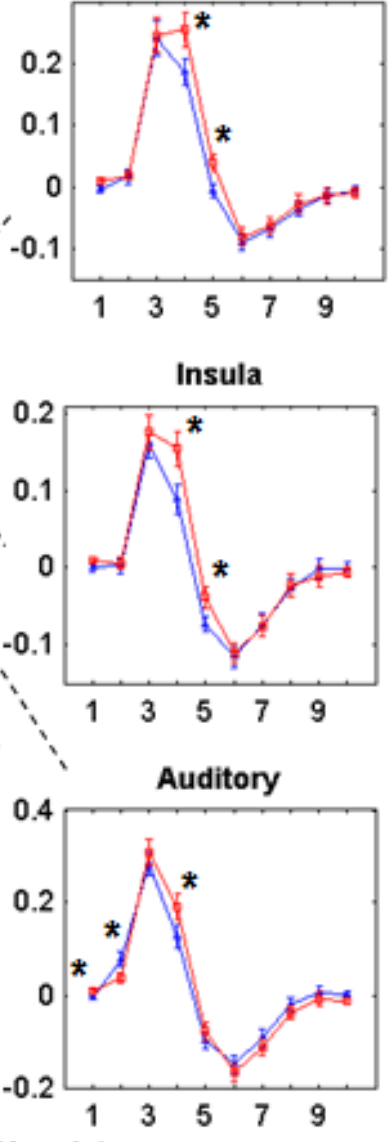

pSMFC

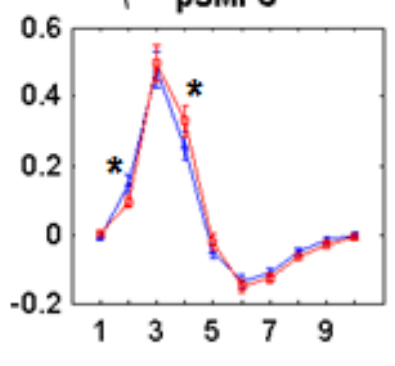

Cingulate

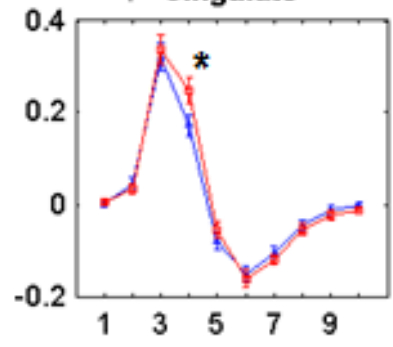

Number of participants in overlay 
Figure 3. Overlay map of all ROls for all participants $(n=20)$ extracted by leave-one-subject-out approach. The vertices in the maps are colored based on the number participants for whom a vertex was included in ROls. Subplots show the mean and standard error of mean (SE) of betavalues for FIR time bins in short (red) and long (blue) SOA conditions. Dashed lines connect the subplots to corresponding ROIs. Stars indicate the FIR time bins in which the mean beta-value significantly differed across the short and long SOA conditions $(p<0.05$, corrected for multiple comparisons). Abbreviations: IFG = inferior frontal gyrus, $\mathrm{pSMFC}=$ posterior superior medial frontal cortex, dMFC = dorsal medial frontal cortex, IFJ= inferior frontal junction, IPG = inferior parietal gyrus, CS-inferior = central sulcus inferior part. $L=$ left hemisphere, $R=$ right hemisphere.

Table 1. List of ROls that were extracted by leave-one-subject-out approach for all participants. BA denotes the Brodmann area, VN denotes the number of vertices in each ROI. All p-values are corrected for multiple comparisons across regions for the main effect and the interaction using FDR at $q<0.05$.

\begin{tabular}{|c|c|c|c|c|c|c|c|c|c|c|}
\hline \multirow[t]{2}{*}{ Region Name } & \multirow[t]{2}{*}{ hemi } & \multicolumn{3}{|c|}{ MIN coordinate } & \multirow[t]{2}{*}{ BA } & \multirow[t]{2}{*}{ NV } & \multicolumn{2}{|c|}{ SOA } & \multicolumn{2}{|c|}{ SOA x Time } \\
\hline & & $x$ & $y$ & $\mathbf{z}$ & & & $F$ & $p$ & $F$ & $p$ \\
\hline \multirow[t]{2}{*}{ Visual } & $\mathbf{L}$ & 2 & -80 & 3 & 17,18 & 8597 & 23.51 & 0.002 & 17.11 & $<0.001$ \\
\hline & $\mathbf{R}$ & 17 & -69 & 13 & & & & & & \\
\hline \multirow[t]{2}{*}{ Auditory } & $\mathbf{L}$ & 54 & -28 & 6 & 42 & 1485 & 0.001 & 0.973 & 11.78 & $<0.001$ \\
\hline & $\mathbf{R}$ & 55 & -24 & 7 & & & & & & \\
\hline \multirow[t]{2}{*}{ IFG } & $\mathbf{L}$ & 33 & 27 & -12 & 44 & 1541 & 6.217 & 0.103 & 8.03 & $<0.001$ \\
\hline & $\mathbf{R}$ & 41 & 14 & 7 & & & & & & \\
\hline \multirow[t]{2}{*}{ Insula } & $\mathbf{L}$ & 26 & 22 & -10 & - & 2787 & 5.446 & 0.109 & 10.5 & $<0.001$ \\
\hline & $\mathbf{R}$ & 44 & -70 & -5 & & & & & & \\
\hline \multirow[t]{2}{*}{ Cingulate } & $\mathbf{L}$ & 11 & 20 & 26 & 6,24 & 3621 & 0.99 & 0.593 & 11.21 & $<0.001$ \\
\hline & $\mathbf{R}$ & 11 & 47 & 32 & & & & & & \\
\hline \multirow[t]{2}{*}{ pSMFC } & $\mathbf{L}$ & 10 & -3 & 56 & 6,8 & 2684 & 0.679 & 0.923 & 12.13 & $<0.001$ \\
\hline & $\mathbf{R}$ & 24 & -26 & 51 & & & & & & \\
\hline \multirow[t]{2}{*}{ Precentral } & $\mathbf{L}$ & 62 & -26 & -11 & 4,6 & 3033 & 0.127 & 0.923 & 12.88 & $<0.001$ \\
\hline & $\mathbf{R}$ & 30 & -11 & 56 & & & & & & \\
\hline \multirow[t]{2}{*}{ Postcentral } & $\mathbf{L}$ & 44 & 33 & 1 & $1,2,3$ & 4875 & 0.023 & 0.947 & 17.55 & $<0.001$ \\
\hline & $\mathbf{R}$ & 40 & -61 & 10 & & & & & & \\
\hline dMFC & $\mathbf{L}$ & 30 & 37 & 16 & 46 & 1855 & 6.866 & 0.103 & 6.519 & $<0.001$ \\
\hline IFJ & $\mathbf{L}$ & 34 & 26 & -9 & 6 & 2084 & 1.219 & 0.593 & 7.887 & $<0.001$ \\
\hline Supramarginal & $\mathbf{L}$ & 59 & -11 & -1 & 40 & 4893 & 3.288 & 0.240 & 11.16 & $<0.001$ \\
\hline IPG & $\mathbf{L}$ & 59 & -10 & -1 & 39 & 909 & 0.804 & 0.593 & 8.737 & $<0.001$ \\
\hline CS-inferior & $\mathbf{L}$ & 38 & 28 & 2 & $1,2,3$ & 689 & 0.052 & 0.947 & 5.302 & $<0.001$ \\
\hline
\end{tabular}

Abbreviations: IFG = inferior frontal gyrus, $\mathrm{pSMFC}=$ posterior superior medial frontal cortex, $\mathrm{dMFC}=$ dorsal medial frontal cortex, IFJ= inferior frontal junction, IPG = inferior parietal gyrus, CS-inferior = central sulcus inferior part. $L=$ left hemisphere, $R=$ right hemisphere. 
Table 3.2. Pairwise comparison of mean activities between the short and long SOA conditions for each time bin and each region. All p-values are corrected for multiple comparisons across time bins using FDR at $q<0.05$.

\begin{tabular}{lllllllll}
\hline Regions name & & \multicolumn{7}{c}{ FIR time points } \\
& & $\mathbf{1}$ & $\mathbf{2}$ & $\mathbf{3}$ & $\mathbf{4}$ & $\mathbf{5}$ & $\mathbf{6}$ & $\mathbf{7}$ \\
\hline Visual & $t$ & 3.395 & 3.401 & 2.827 & 6.089 & 6.297 & 1.125 & -1.094 \\
Auditory & $p$ & $\mathbf{0 . 0 1 7}$ & $\mathbf{0 . 0 1 7}$ & $\mathbf{0 . 0 4 2}$ & $\mathbf{0 . 0 0 0}$ & $\mathbf{0 . 0 0 0}$ & 0.412 & 0.415 \\
& $t$ & 3.750 & -4.121 & 1.767 & 5.322 & 1.519 & -1.593 & -2.388 \\
IFG & $p$ & $\mathbf{0 . 0 1 0}$ & $\mathbf{0 . 0 0 5}$ & 0.196 & $<\mathbf{0 . 0 0 1}$ & 0.268 & 0.242 & 0.087 \\
& $t$ & 1.825 & 0.197 & 0.318 & 5.698 & 3.863 & 0.909 & 0.776 \\
Insula & $p$ & 0.187 & 0.890 & 0.825 & $<\mathbf{0 . 0 0 1}$ & $\mathbf{0 . 0 0 8}$ & 0.509 & 0.591 \\
& $t$ & 1.823 & 0.455 & 1.279 & 6.271 & 3.170 & 0.304 & -0.207 \\
Cingulate & $p$ & 0.187 & 0.751 & 0.352 & $<\mathbf{0 . 0 0 1}$ & $\mathbf{0 . 0 2 1}$ & 0.826 & 0.889 \\
& $t$ & 0.531 & -1.333 & 1.401 & 5.713 & 2.143 & -1.056 & -1.763 \\
pSMFC & $p$ & 0.720 & 0.334 & 0.306 & $<\mathbf{0 . 0 0 1}$ & 0.120 & 0.435 & 0.196 \\
& $t$ & 1.166 & -3.992 & 1.309 & 5.061 & 2.278 & -1.105 & -1.716 \\
Precentral & $p$ & 0.401 & $\mathbf{0 . 0 0 6}$ & 0.342 & $\mathbf{0 . 0 0 1}$ & 0.097 & 0.412 & 0.208 \\
& $t$ & 1.811 & -3.617 & -0.012 & 5.386 & 3.170 & -1.261 & -2.500 \\
Postcentral & $p$ & 0.188 & $\mathbf{0 . 0 1 3}$ & 0.990 & $<\mathbf{0 . 0 0 1}$ & $\mathbf{0 . 0 2 1}$ & 0.354 & 0.076 \\
& $t$ & 2.201 & -3.467 & 1.749 & 6.374 & 2.437 & -2.518 & -3.328 \\
L DMFC & $p$ & 0.111 & $\mathbf{0 . 0 1 7}$ & 0.199 & $<\mathbf{0 . 0 0 1}$ & 0.083 & 0.076 & $\mathbf{0 . 0 1 8}$ \\
& $t$ & 0.611 & 0.612 & 1.854 & 4.217 & 3.406 & 0.725 & 0.300 \\
L IFJ & $p$ & 0.668 & 0.668 & 0.182 & $\mathbf{0 . 0 0 4}$ & $\mathbf{0 . 0 1 7}$ & 0.613 & 0.826 \\
& $t$ & 2.104 & -2.317 & -0.331 & 5.442 & 2.872 & -0.120 & -0.645 \\
L Supramarginal & $p$ & 0.126 & 0.095 & 0.820 & $<\mathbf{0 . 0 0 1}$ & $\mathbf{0 . 0 3 9}$ & 0.925 & 0.659 \\
& $t$ & 3.365 & 0.727 & -0.174 & 5.531 & 3.243 & 0.155 & -1.111 \\
L IPG & $p$ & $\mathbf{0 . 0 1 8}$ & 0.613 & 0.903 & $<\mathbf{0 . 0 0 1}$ & $\mathbf{0 . 0 2 1}$ & 0.911 & 0.412 \\
& $t$ & -0.495 & 5.655 & 3.173 & -1.616 & -2.606 & -2.508 & -2.047 \\
L CS-inferior & $p$ & 0.743 & $<\mathbf{0 . 0 0 1}$ & $\mathbf{0 . 0 2 1}$ & 0.238 & 0.066 & 0.076 & 0.130 \\
& $t$ & 2.089 & 5.823 & 0.666 & 0.393 & 0.617 & -1.414 & -1.452 \\
& $p$ & 0.126 & $<\mathbf{0 . 0 0 1}$ & 0.648 & 0.789 & 0.668 & 0.305 & 0.293 \\
\hline
\end{tabular}

Abbreviations: IFG = inferior frontal gyrus, pSMFC = posterior superior medial frontal cortex, $\mathrm{dMFC}=$ dorsal medial frontal cortex, IFJ= inferior frontal junction, IPG = inferior parietal gyrus, CS-inferior $=$ central sulcus inferior part. $L=$ left hemisphere.

\section{Multivariate pattern analysis:}

To compare the driving task decoding accuracy in short and long SOAs, we followed a leave-one-subject-out approach and a union analysis, to extract ROIs from the clusters that decode the driving task above chance level in either the short or the long SOA conditions for the FIR time bin 3. The mean accuracy of the ROls was then compared for two SOA conditions in FIR time bins 2 to 5 . Figure 4 shows an overlay of the ROls of all 
participants. Four main regions can be seen in Figure 4 including the left and right visual cortex, right superior parietal lobe (SPL) and right motor cortex. First, we ran a two-way repeated-measure ANOVA with SOA and FIR time bins as two factors to compare the accuracy for each ROI in two SOA across FIR time bins 2 to 5 (Figure 3, panel B). The details of statistical tests can be seen in table 3. The main effect of SOA was not significant in any of the ROls ( $p s>0.05)$. The interaction of SOA and time bins was significant for the right SPL $(F(3,57)=9.32, p<0.0001$, two-way repeated measures ANOVA).

Focusing on individual time bins in the SPL region, we next compared the accuracy for each time bin in each condition to chance level. The accuracy for decoding the driving direction was above chance level in the third and fourth time bins in the long SOA condition (ts $>6.01, p s<0.001$, paired t-test) and was not significantly greater from chance in any of the time bins in the short SOA condition ( $p s>0.05)$. Further pairwise comparisons between short and long SOA conditions in each time bin showed that the decoding the driving direction for long SOA was significantly higher than the short SOA in time bins third and fourth for SPL ( $t s>3.02$, ps $<0.014$, paired t-test, see table 4). These results show that although the amount of information about the driving task does not change in the visual and motor regions, it decreases significantly in the SPL region. Furthermore, a Pearson correlation analysis revealed a negative correlation between the turn direction decoding accuracy in the SPL region and the reaction time of the driving task obtained from the behavioral results $(r=-0.507, p=0.032$, corrected for multiple comparisons). This correlation was not significant for the other three regions (Figure 3, panel c). The drop of information in the SPL region could play a role in dual-task 
interference and the drop in performance when the two tasks are performed concurrently.

Our further exploration in data cleared that removing the error trials did not qualitatively change the results.

A

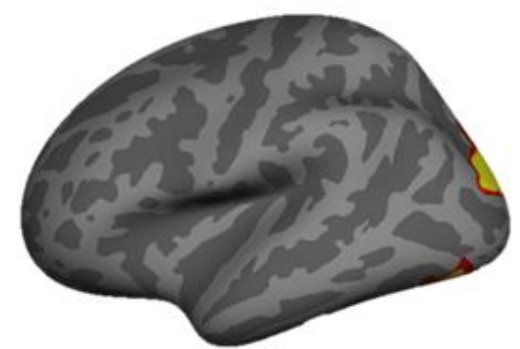

visual

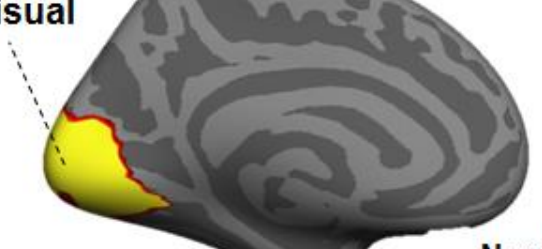

Number of participants in overlay

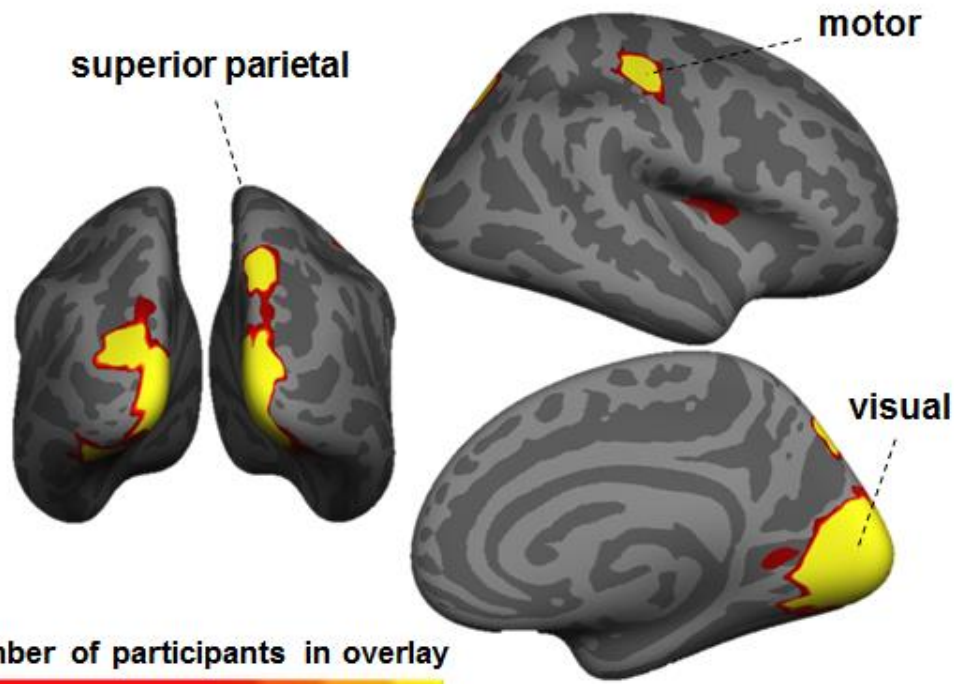

1

20

B

L visual

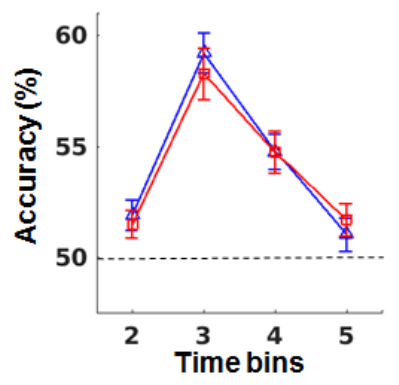

C

$r=-0.018, p=0.911$

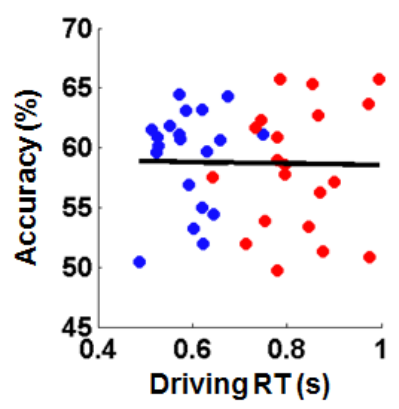

R visual

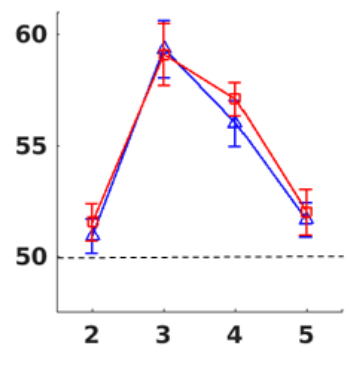

$r=-0.072, p=0.873$

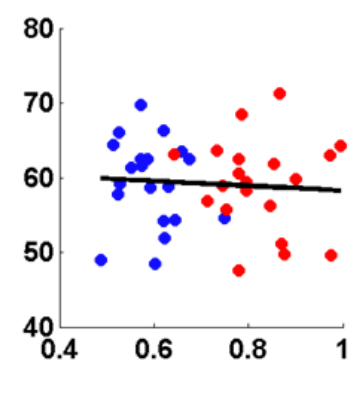

R superior

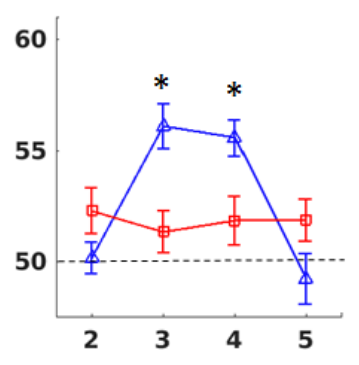

$r=-0.507, p=0.032$ *

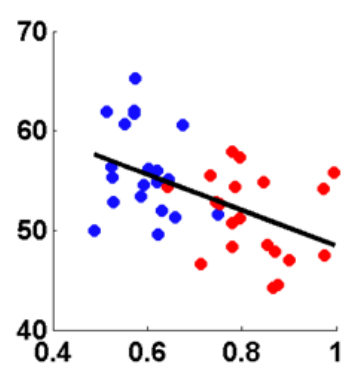

R motor

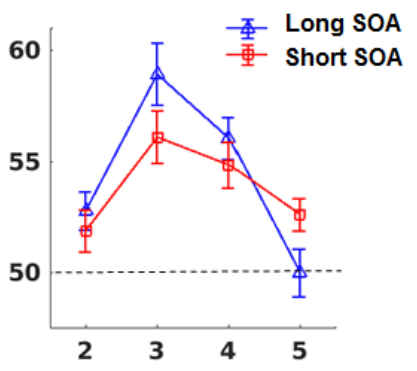

$r=-0.330, p=0.074$

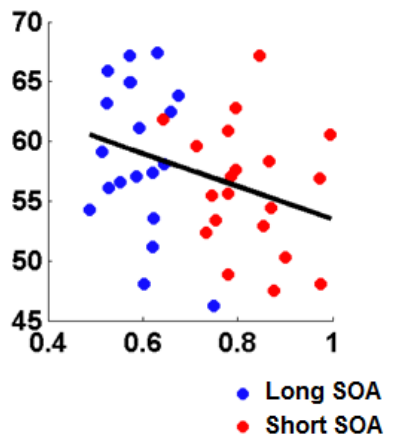


Figure 4. A) Overlay of ROls for all participants $(n=20)$ for the decoding of driving direction. The vertices in the maps are colored based on the number participants for whom a vertex was included in ROIs. Dashed lines connect the subplots to corresponding ROls. B) Subplots show the mean and SE of driving direction decoding accuracy for FIR time bins 2-5 for short (red) and long (blue) SOA conditions extracted ROls. Dashed lines show the chance level (50\%). Stars indicate the FIR time bins in which accuracy significantly differed across the short and long SOA conditions ( $p<0.05$, corrected for multiple comparisons). C) The Pearson correlation between the decoding accuracy of the driving direction and behavioral reaction time of driving direction for ROls. Abbreviations: $L=$ Left hemisphere, $R=$ Right hemisphere.

Table 3.3. Two-way repeated-measure ANOVA for the effect of SOA and time on accuracies in each ROI. BA denotes the Brodmann area, VN denotes the number of vertices in each ROI. All p-values were corrected for multiple comparisons across ROls using FDR at $q<0.05$, significant p-values are shown in bold text.

\begin{tabular}{|c|c|c|c|c|c|c|c|c|c|}
\hline \multirow[t]{2}{*}{ Region names } & \multirow[t]{2}{*}{ BA } & \multicolumn{3}{|c|}{ MNI coordinate } & \multirow[t]{2}{*}{ NV } & \multicolumn{2}{|c|}{ SOA } & \multicolumn{2}{|c|}{ SOA x Time } \\
\hline & & $\mathbf{x}$ & $\mathbf{y}$ & $\mathbf{z}$ & & $\mathbf{F}$ & $\mathbf{p}$ & $\mathbf{F}$ & $\mathbf{p}$ \\
\hline L Visual cortex & 17,18 & -8 & -92 & 7 & 6663 & 0.147 & 0.704 & 0.649 & 0.782 \\
\hline R Visual cortex & 17,18 & 11 & -76 & 5 & 5560 & 0.437 & 0.516 & 0.308 & 0.819 \\
\hline R Motor cortex & 1,3 & 46 & -17 & 52 & 1200 & 0.578 & 0.456 & 3.460 & 0.051 \\
\hline $\begin{array}{l}\text { R Superior } \\
\text { parietal lobe }\end{array}$ & 7 & 15 & -71 & 46 & 925 & 1.970 & 0.176 & 9.327 & $<0.001$ \\
\hline
\end{tabular}

Abbreviations: $L=$ left hemisphere, $R=$ right hemisphere. 
Table 3.4. Comparison of accuracy with chance level (50\%) for SOA conditions and pairwise comparison of accuracies between the short and long SOA conditions for each time bin and each region. All $p$-values were corrected for multiple comparisons across time bins using FDR at $q<$ 0.05. Significant $p$-values are shown in bold text.

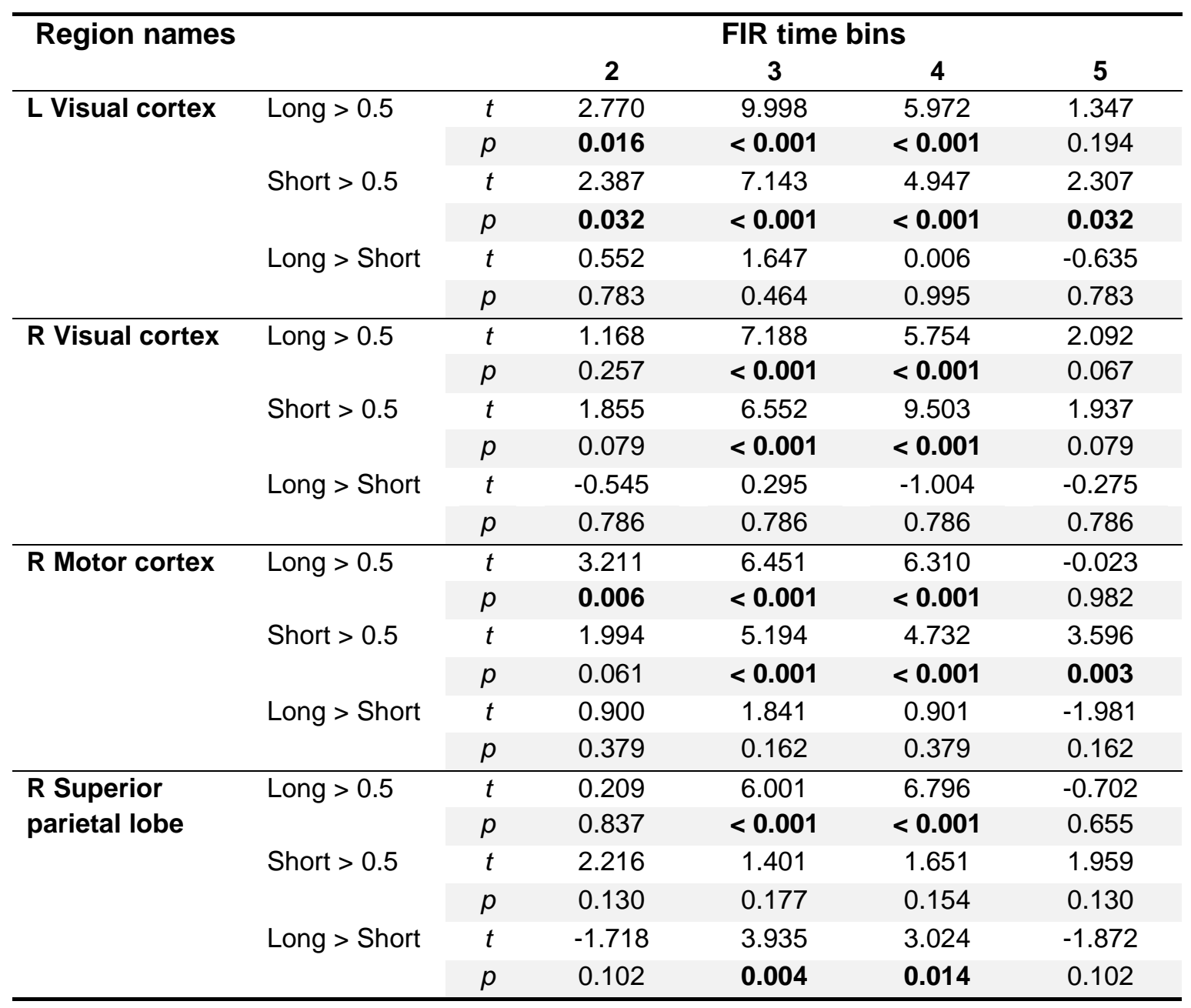

Abbreviations: $L=$ left hemisphere, $R=$ right hemisphere.

\section{Discussion}

Here, we investigated the effect of dual-task interference on the BOLD response in a dualtask paradigm in which participants performed a driving turn in a simulated driving environment along with a tone discrimination task. The two tasks were either presented close together (short SOA) or far from each other (long SOA) in time. The behavioral results showed an increase in $\mathrm{RT}$ and a decrease in accuracy of the driving task in the 
short SOA compared to the long SOA trials consistent with previous studies (Hibberd et al., 2013; Levy et al., 2006), confirming that the performance is influenced by dual-task interference. We performed a time-resolved $\mathrm{fMRI}$ analysis by investigating the mean and patterns of brain activity at multiple time bins after the stimulus onset. In the univariate analysis we showed that although the peak of the hemodynamic response is at the same time point for both short and long SOA conditions, in the short SOA condition, the hemodynamic response drops with a gentler slope compared to the long SOA condition. This effect was observed in a large swath of brain regions including sensory, decisionrelated, and motor regions. The results of multivariate pattern analysis, revealed regions that carry information about the driving direction including superior parietal lobe (SPL), visual and motor regions. Comparing the decoding accuracies across short and long SOA conditions, we showed for the first time that the information for the driving direction gets disrupted by dual-task interference in the right SPL but not in the visual and motor regions.

Although we observed a modulation in the BOLD response in numerous brain regions across SOA conditions, only a few regions including the bilateral visual cortex, right motor cortex, and right SPL carried information for the driving direction. In other words, the accuracy for decoding the driving direction was significantly above chance level only in those three regions. Further investigations revealed that the accuracy for decoding the driving direction was not different across SOA conditions in the visual and motor cortices, but it significantly decreased in the short compared to the long SOAs in the SPL. In addition, the brain-behavior correlations also showed that there was a negative correlation between the reaction time of the driving turn and the decoding accuracy of the driving direction in the SPL region. These findings imply that the information for the driving 
direction is disturbed in the SPL during dual-task interference. To the best of our knowledge, this finding is novel and has not been reported in previous studies.

A recent Magnetoencephalography (MEG) study by Marti, King, and Dehaene (2015), showed that the information is disturbed during the central processing stage (about 200$400 \mathrm{~ms}$ after the onset of the stimulus). However, the location of the distortion of information was not precisely clear due to limitation in the spatial resolution of MEG signal. The findings of the current study reveal that the source of disturbance of information during the dual-task interference could be the SPL region.

Even though it is not possible to pinpoint the exact cause of this decline in classification accuracy in the SPL region from the current study, we may speculate about its possible sources. On the one hand, the SPL region found in our study overlaps with a region suggested to be the holomologe of Lateral Intraparietal (LIP) in non-human primates (Grefkes \& Fink, 2005) that is involved in evidence accumulation for visual perceptual decision making (Shadlen \& Newsome, 2001). The reduced classification accuracies in the SPL region during dual-task interference, therefore, could be related to the disturbance in the process of decision making for the driving turn. This alternative is in line with recent behavioral modeling studies (Abbas-Zadeh et al., 2019; Zylberberg et al., 2012) that have suggested changes in the decision processes during dual-task interference.

On the other hand, SPL region has been implicated in maintaining information during visual working memory (Rowe et al., 2000; Xu \& Chun, 2006) and contains more information about an attended compared to an unattended item (Vaziri-Pashkam \& Xu, 
2017). Considering these findings, another possibility for the drop in classification accuracy in the SPL could be the impairment in the maintenance of the information for the driving direction due to the distractions caused by the secondary task. Future studies could elucidate processes that lead to the drop in classification accuracy in SPL.

The univariate analysis indicated a significant interaction between SOA and time bins on the BOLD response in the frontal regions such as inferior frontal gyrus, inferior frontal junction, dorsal medial frontal cortex, superior medial frontal cortex, insula, anterior cingulate, and middle cingulate cortex. In these regions a change in the fall of HRF (an increase in the activation of the fourth and fifth-time bins) was observed in the short SOA compared to the long SOA conditions. No difference was found in the rise and peak of the HRF across SOA conditions in these regions. Several previous dual-task studies that have examined the changes in the BOLD signal, during dual-task interference, have reported an increase in the activation in the frontal regions (Erickson et al., 2005; Jiang et al., 2004; Nijboer et al., 2014; Sigman \& Dehaene, 2008; Stelzel, Brandt, \& Schubert, 2009). These studies have not investigated the changes in the shape of HRF across SOAs. Our results extend these findings to show the changes in the shape of HRF suggesting that the increase of activity in these regions may have resulted from the later fall of the HRF during dual-task interference.

A few studies have investigated the changes in the shape of HRF during dual-task interference using artificial dual-task paradigms (Dux et al., 2006; Dux et al., 2009; Tombu et al., 2011). These studies have shown a modulation in the shape of HRF in the frontal and parietal regions, although they have only focused on specific fronto-parietal regions and have not investigated the changes in the shape of HRF in sensory and motor regions. 
Unlike these studies, here, we used a well-controlled lane change driving scenario close to real-world dual-task conditions and observed similar modulations in the HRF shape in the sensory, central and motor regions.

The univariate analysis revealed clear signs of the change in the shape of HRF in sensory cortices during dual-task interference. Nevertheless, the information for the driving direction was not significantly affected in the visual cortex. The increase of activity of sensory regions in the short compared to the long SOA might be due to top-down feedback signals to sensory regions to keep these regions active for longer durations and increase their activity in order to compensate for the noise caused by dual-task interference.

Our results also indicated a change in the shape (a slow rise and a shallow slope in the fall) of HRF in motor regions without any change in the information content for driving direction. These changes in the fMRI signal might be related to a delay in the processing of the motor stage during dual-task interference. These results are novel and to the best of our knowledge have not been reported in previous fMRI studies of dual-task interference. The delays in the motor stage of processing have been proposed in previous behavioral modeling studies (Zylberberg et al., 2012) and are in line with our previous behavioral and modeling findings (Abbas-Zadeh et al., 2019).

Taken together, the results from our univariate and multivariate analyses are complementary. Our multivariate analysis, looking at classification accuracy for the driving direction, reveals regions that are specific for the individual tasks (in this case the driving turn) as it carves out the information content of each region for a particular sensory 
input, decision, or motor output. The univariate analysis, on the other hand, can uncover regions that are implicated in domain general processes such as cognitive control and task management. In fact, our study demonstrates that the regions found in the analyses are complementary. Frontal regions show changes in univariate response across SOAs but do not carry information about the driving task suggesting their involvement in domain general processes. On the other hand, the SPL region shows changes in classification accuracy, with no difference in univariate response across SOA, suggesting its involvement in information processing of the driving turn. A comprehensive understanding of the neural correlates of dual-task interference thus requires gathering evidence from both univariate and multivariate analyses.

Taken together, we used a controlled paradigm to study dual-task interference in a realistic setup to investigate the behavioral and neural signatures of dual-task interference. The performance of the driving and the secondary task was influenced by dual-task interference. The shape of HRF was modulated by SOA in sensory, decisionrelated, and motor regions. The information content for driving direction did not significantly change in the sensory and motor regions, but it decreased in the SPL in short compared to long SOA trials. These results extended our understanding of the neural correlates of dual-task interference and are informative for formulating biologically plausible models of dual-task interference. They also have potential applications in improving the quality of driving behavior and reducing the number of accidents caused by driver distraction.

\section{Acknowledgements}


We thank Mahdi Shafiei for his assistance in developing the simulated driving environment. This research was supported in part by the Intramural Research Program of the $\mathrm{NIH}$, National Institute of Mental Health (ZIA MH002035-39). The collection and analysis of the data was performed entirely at the Institute for Research in Fundamental Sciences (IPM) supported by a research grant from IPM.

\section{References}

Abbas-Zadeh, M., Hossein-Zadeh, G.-A., \& Vaziri-Pashkam, M. (2019). Dual-task Interference in a Simulated Driving Environment: Serial or Parallel Processing? bioRxiv, 853119. doi:10.1101/853119

Al-Hashimi, O., Zanto, T. P., \& Gazzaley, A. (2015). Neural sources of performance decline during continuous multitasking. cortex, 71, 49-57.

Benjamini, Y., \& Hochberg, Y. (1995). Controlling the false discovery rate: a practical and powerful approach to multiple testing. Journal of the Royal statistical society: series $B$ (Methodological), 57(1), 289-300.

Cox, D. D., \& Savoy, R. L. (2003). Functional magnetic resonance imaging (fMRI) "brain reading": detecting and classifying distributed patterns of fMRI activity in human visual cortex. Neuroimage, 19(2 Pt 1), 261-270. doi:10.1016/s1053-8119(03)00049-1

Dale, A. M. (1999). Optimal experimental design for event-related fMRI. Human brain mapping, 8(2-3), 109-114.

Dale, A. M., Fischl, B., \& Sereno, M. I. (1999). Cortical surface-based analysis: I. Segmentation and surface reconstruction. Neuroimage, 9(2), 179-194.

Dux, P. E., Ivanoff, J., Asplund, C. L., \& Marois, R. (2006). Isolation of a central bottleneck of information processing with time-resolved fMRI. Neuron, 52(6), 1109-1120.

Dux, P. E., Tombu, M. N., Harrison, S., Rogers, B. P., Tong, F., \& Marois, R. (2009). Training improves multitasking performance by increasing the speed of information processing in human prefrontal cortex. Neuron, 63(1), 127-138.

Erickson, K. I., Colcombe, S. J., Wadhwa, R., Bherer, L., Peterson, M. S., Scalf, P. E., \& Kramer, A. F. (2005). Neural correlates of dual-task performance after minimizing task-preparation. Neuroimage, 28(4), 967-979.

Esterman, M., Tamber-Rosenau, B. J., Chiu, Y.-C., \& Yantis, S. (2010). Avoiding nonindependence in fMRI data analysis: leave one subject out. Neuroimage, 50(2), 572-576.

Evans, A. C., Collins, D. L., Mills, S., Brown, E., Kelly, R., \& Peters, T. M. (1993). 3D statistical neuroanatomical models from $305 \mathrm{MRI}$ volumes. Paper presented at the Nuclear Science Symposium and Medical Imaging Conference, 1993., 1993 IEEE Conference Record.

Grefkes, C., \& Fink, G. R. (2005). The functional organization of the intraparietal sulcus in humans and monkeys. Journal of anatomy, 207(1), 3-17.

Henson, R., Rugg, M. D., \& Friston, K. J. (2001). The choice of basis functions in event-related fMRI. Neurolmage, 13(6), 149-149.

Herath, Klingberg, Young, Amunts, \& Roland. (2001). Neural correlates of dual task interference can be dissociated from those of divided attention: an fMRI study. Cerebral cortex, 11(9), 796-805. 
Hesselmann, G., Flandin, G., \& Dehaene, S. (2011). Probing the cortical network underlying the psychological refractory period: a combined EEG-fMRI study. Neuroimage, 56(3), 16081621.

Hibberd, D. L., Jamson, S. L., \& Carsten, O. M. (2013). Mitigating the effects of in-vehicle distractions through use of the Psychological Refractory Period paradigm. Accident Analysis \& Prevention, 50, 1096-1103.

Jiang, Y., Saxe, R., \& Kanwisher, N. (2004). Functional magnetic resonance imaging provides new constraints on theories of the psychological refractory period. Psychological Science, 15(6), 390-396.

Just, M. A., Keller, T. A., \& Cynkar, J. (2008). A decrease in brain activation associated with driving when listening to someone speak. Brain research, 1205, 70-80.

Kamitani, Y., \& Tong, F. (2005). Decoding the visual and subjective contents of the human brain. Nature neuroscience, 8(5), 679-685.

Kriegeskorte, N., Simmons, W. K., Bellgowan, P. S., \& Baker, C. I. (2009). Circular analysis in systems neuroscience: the dangers of double dipping. Nature neuroscience, 12(5), 535.

Levy, J., Pashler, H., \& Boer, E. (2006). Central interference in driving: Is there any stopping the psychological refractory period? Psychological Science, 17(3), 228-235.

Loose, L. S., Wisniewski, D., Rusconi, M., Goschke, T., \& Haynes, J.-D. (2017). Switchindependent task representations in frontal and parietal cortex. Journal of Neuroscience, 37(33), 8033-8042.

Marois, R., \& Ivanoff, J. (2005). Capacity limits of information processing in the brain. Trends in cognitive sciences, 9(6), 296-305.

Marti, S., King, J.-R., \& Dehaene, S. (2015). Time-resolved decoding of two processing chains during dual-task interference. Neuron, 88(6), 1297-1307.

Nijboer, M., Borst, J., van Rijn, H., \& Taatgen, N. (2014). Single-task fMRI overlap predicts concurrent multitasking interference. Neurolmage, 100, 60-74.

Oosterhof, N. N., Connolly, A. C., \& Haxby, J. V. (2016). CoSMoMVPA: multi-modal multivariate pattern analysis of neuroimaging data in Matlab/GNU Octave. Frontiers in neuroinformatics, 10, 27.

Oosterhof, N. N., Wiggett, A. J., Diedrichsen, J., Tipper, S. P., \& Downing, P. E. (2010). Surfacebased information mapping reveals crossmodal vision-action representations in human parietal and occipitotemporal cortex. Journal of neurophysiology, 104(2), 1077-1089.

Pashler, H. (1994). Dual-task interference in simple tasks: data and theory. Psychological bulletin, 116(2), 220.

Rowe, J. B., Toni, I., Josephs, O., Frackowiak, R. S., \& Passingham, R. E. (2000). The prefrontal cortex: response selection or maintenance within working memory? Science, 288(5471), 1656-1660.

Shadlen, M. N., \& Newsome, W. T. (2001). Neural basis of a perceptual decision in the parietal cortex (area LIP) of the rhesus monkey. Journal of neurophysiology, 86(4), 1916-1936.

Sigman, M., \& Dehaene, S. (2008). Brain mechanisms of serial and parallel processing during dual-task performance. Journal of Neuroscience, 28(30), 7585-7598.

Stelzel, C., Brandt, S. A., \& Schubert, T. (2009). Neural mechanisms of concurrent stimulus processing in dual tasks. Neuroimage, 48(1), 237-248.

Tombu, M. N., Asplund, C. L., Dux, P. E., Godwin, D., Martin, J. W., \& Marois, R. (2011). A unified attentional bottleneck in the human brain. Proceedings of the National Academy of Sciences, 108(33), 13426-13431.

Vaziri-Pashkam, M., \& Xu, Y. (2017). Goal-directed visual processing differentially impacts human ventral and dorsal visual representations. Journal of Neuroscience, 37(36), 8767-8782.

$\mathrm{Xu}, \mathrm{Y}$. , \& Chun, M. M. (2006). Dissociable neural mechanisms supporting visual short-term memory for objects. Nature, 440(7080), 91-95. 
bioRxiv preprint doi: https://doi.org/10.1101/2020.07.28.224394; this version posted July 29, 2020. The copyright holder for this preprint (which

was not certified by peer review) is the author/funder, who has granted bioRxiv a license to display the preprint in perpetuity. It is made available under aCC-BY-NC-ND 4.0 International license.

Zylberberg, A., Ouellette, B., Sigman, M., \& Roelfsema, P. R. (2012). Decision making during the psychological refractory period. Current biology, 22(19), 1795-1799. 